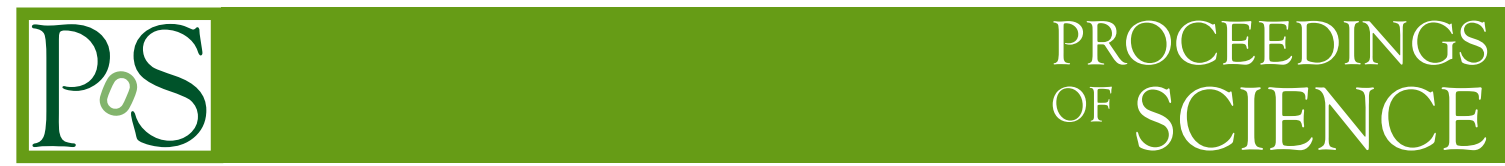

\title{
Overview of recent results from $\mathrm{LHCb}$
}

\section{Sajan Easo*}

STFC, Rutherford-Appleton Laboratory,U.K., On behalf of the $\mathrm{LHCb}$ collaboration

E-mail: Sajan.Easo@CERN.CH

An overview of the LHCb experiment is presented. Recent results from this experiment on $\mathrm{CP}$ violation in beauty and charm decays are reported.

The XXI International Workshop High Energy Physics and Quantum Field Theory, June 23 to June 30, 2013

Saint Petersburg Area, Russia

${ }^{*}$ Speaker. 


\section{Introduction}

The $\mathrm{LHCb}$ detector [5] is a single-arm forward spectrometer covering the pseudo rapidity range $2<\eta<5$ and is designed for the study of particles containing $b$ or $c$ quarks. The main goal of the experiment is to look for signals beyond the standard model in particle physics. It also makes various measurements related to electroweak physics and QCD.

At LHC the production of $b \bar{b}$ is peaked in the forward and backward directions. LHCb measurements show that

$\sigma(p p)=59.9 \pm 2.7 \mathrm{mb}[1]$ and that

$\sigma(p p \rightarrow b \bar{b} X)=(75.3 \pm 5.4$ (stat) \pm 13.0 (syst) $) \mu \mathrm{b}$ for $2<\eta<6[2]$

$\sigma(p p \rightarrow c \bar{c} X)=(1419 \pm 12$ (stat) \pm 116 (syst) \pm 65 (frag) $) \mu \mathrm{b}$ for $2.0<\eta<4.5$ and $P_{T}<8 \mathrm{GeV} / \mathrm{c}$. [3]

This results in a large production of $b$-hadrons and $c$-hadrons whose decays can be studied in LHCb. In $2011 \mathrm{LHCb}$ collected $1 \mathrm{fb}^{-1}$ of data and in 2012 it collected $2 \mathrm{fb}^{-1}$ of data. It was designed to collect the data at a luminosity of $2 \times 10^{32} \mathrm{~cm}^{-2} \mathrm{~s}^{-1}$, but the average luminosity in 2011 was $3.2 \times 10^{32} \mathrm{~cm}^{-2} \mathrm{~s}^{-1}$ and that in 2012 was $3.9 \times 10^{32} \mathrm{~cm}^{-2} \mathrm{~s}^{-1}$. This resulted in detector occupancies larger than those foreseen from the design, but the data were taken successfully without jeopardizing the physics output.

The analysis of the data from LHCb are aimed at multiple objectives. One is to measure $\mathrm{CP}$ violation in different channels and look for any inconsistencies that may give a hint on the existence new physics. Another objective is to search for rare decays and from the different branching fractions and other parameters obtained, look for signals of new physics. In this paper, recent results from the $\mathrm{CP}$ violation in $\mathrm{B}$-decays and $\mathrm{D}$-decays are quoted. The results from rare decays are covered in another paper [4] presented at this conference.

In this paper, in section 2 a brief description of the $\mathrm{LHCb}$ detector is given. In section 3 the results on mixing and CP-violation from B-decays are presented and in section 4 the results on mixing and CP-violation from the charm decays are quoted.

\section{LHCb Detector}

The detector includes a high-precision tracking system consisting of a silicon-strip vertex detector surrounding the $p p$ interaction region, a large-area silicon-strip detector located upstream of a dipole magnet with a bending power of about $4 \mathrm{Tm}$, and three stations of silicon-strip detectors and straw drift tubes placed downstream. The combined tracking system provides a momentum measurement with relative uncertainty that varies from $0.4 \%$ at $5 \mathrm{GeV} / \mathrm{c}$ to $0.6 \%$ at $100 \mathrm{GeV} / \mathrm{c}$, and impact parameter resolution of $20 \mu \mathrm{m}$ for tracks with large transverse momentum. Different types of charged hadrons are distinguished by information from two ring-imaging Cherenkov detectors [6]. Photon, electron and hadron candidates are identified by a calorimeter system consisting of scintillating-pad and preshower detectors, an electromagnetic calorimeter and a hadronic calorimeter. Muons are identified by a system composed of alternating layers of iron and multiwire proportional chambers [7]. The trigger [8] consists of a hardware stage, based on information from the calorimeter and muon systems, followed by a software stage, which applies a full event reconstruction. 

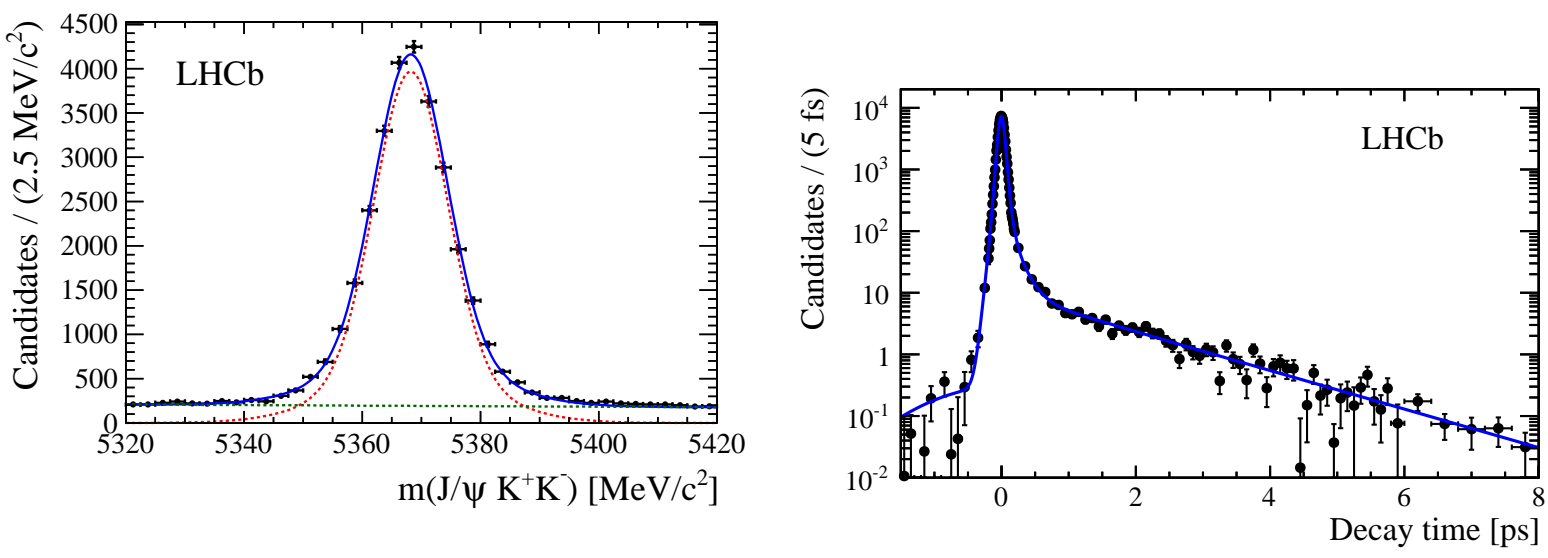

Figure 1: Left: Invariant mass of the $B_{s}^{0} \rightarrow J / \psi K^{+} K^{-}$channel. The mass resolution obtained here is $6 \mathrm{MeV} / \mathrm{c}$. Right: Decay time distribution of $B_{s}^{0} \rightarrow J / \psi K^{+} K^{-}$candidates. The decay model has a delta function for prompt $\mathrm{J} / \psi$ component and two exponential functions for the $\mathrm{J} / \psi$ from the $B_{s}$ decay, all of which are convoluted with the resolution in data. The resolution measured from the prompt $\mathrm{J} / \psi$ component is $45 \mathrm{fs}$.

\subsection{Performance of the vertex detector}

Typical primary vertex resolutions measured for 25 tracks are 16 microns and 76 microns in the directions transverse and longitudinal to the beam direction respectively. Figure 1 shows the invariant mass and decay time of $B_{s}$ reconstructed in the $B_{s}^{0} \rightarrow J / \psi K^{+} K^{-}$channel. This shows a mass resolution of $6 \mathrm{MeV} / \mathrm{c}^{2}$ and a decay time resolution of $45 \mathrm{fs}$. Here the decay time resolution is obtained from the prompt $J / \psi$ background. These indicate the excellent vertex resolutions provided by the vertex detector.

\subsection{Performance of the Cherenkov detectors}

The Particle identification (PID) performance [6] of the two Cherenkov detectors are verified using calibration samples where the PID of the particles are determined from the decay kinematics, independent of the information from the RICH. Figure 2 show the kaon identification efficiency as a function of the momentum of the particle for two different cuts of the log-likelihood algorithm used for the PID. The probability to misidentify a pion as kaon is also plotted in this figure. Excellent PID performance can be seen in this figure, for most of the momentum range 1-100 GeV/c.

\section{Results on mixing and $\mathrm{CP}$ violation in B-Decays}

\section{1 $B_{s}$ mixing}

This is determined in the channel $B_{s} \rightarrow D_{s} \pi$ where [9] the $D_{s}$ decays into any of the 5 decay modes $D_{s}^{-} \rightarrow \phi\left(K^{+} K^{-}\right) \pi^{-}, D_{s}^{-} \rightarrow K^{* 0}\left(K^{+} \pi^{-}\right) K^{-}, D_{s}^{-} \rightarrow K^{+} K^{-} \pi^{-}$(non-resonant),$D_{s}^{-} \rightarrow$ $K^{-} \pi^{+} \pi^{-}$and $D_{s}^{-} \rightarrow \pi^{-} \pi^{+} \pi^{-}$. From 34000 candidate events selected from the 2011 data, the $B_{s}$ decay time is fitted to a probability density function (PDF) which is a convolution of a theoretical 


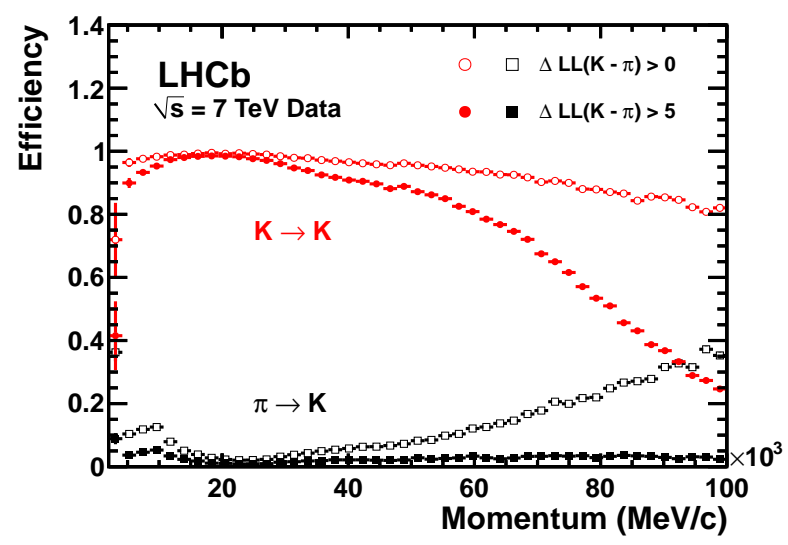

Figure 2: PID performance of the RICH from calibration data. The red points are the kaon identification efficiency and the black points are the probability to misidentify a pion as a kaon. The red empty circles and black empty squares are the efficiency and the misidentification probability for a looser cut of the PID algorithm.

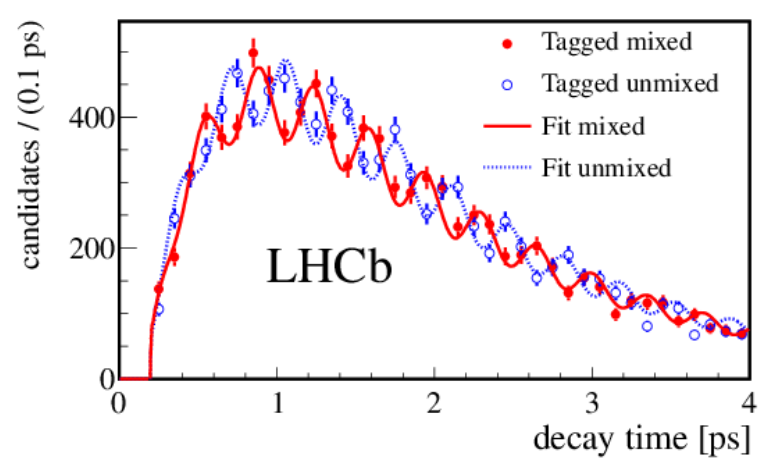

Figure 3: $B_{S}$ decay time in the channel $B_{S} \rightarrow D_{s} \pi$. The red points are for the candidates which are tagged as oscillated (mixed) and the blue points are for the candidates which are tagged as not oscillated (unmixed).

distribution based on the $B_{s}$ decay width, decay time resolution function, decay time acceptance function and the information from flavour tagging. Figure 3 shows the $B_{s}$ decay time distributions for the events where the $B_{s}$ was tagged as oscillated and for the events where the $B_{s}$ was tagged as not oscillated, before decaying. The mixing frequency measured is $17.668 \pm 0.023$ (stat) \pm $0.006(s y s t) \mathrm{ps}^{-1}$. This is the most precise measurement of this quantity to date.

\subsection{CP asymmetry in charmless B-decays}

\subsubsection{CP asymmetry in $B^{0} \rightarrow K^{+} \pi^{-}$and $B_{s}^{0} \rightarrow K^{-} \pi^{+}$}

One can measure direct $\mathrm{CP}$ violation in the channel $B^{0} \rightarrow K^{+} \pi^{-}$and $B_{s} \rightarrow K^{-} \pi^{+}$[10] since their decay amplitudes have contributions from tree and penguin diagrams. This measurement is sensitive to the phase of the CKM element $V_{u b}$ and the CKM angle $\gamma$. In 2011, LHCb collected 41420 candidates in the $B^{0} \rightarrow K^{+} \pi^{-}$channel and 1065 candidates in the $B_{s} \rightarrow K^{-} \pi^{+}$channel. While selecting events in these channels, results from the particle identification was crucial in order identify the kaons and pions in the final states. The raw asymmetry determined contains the true 

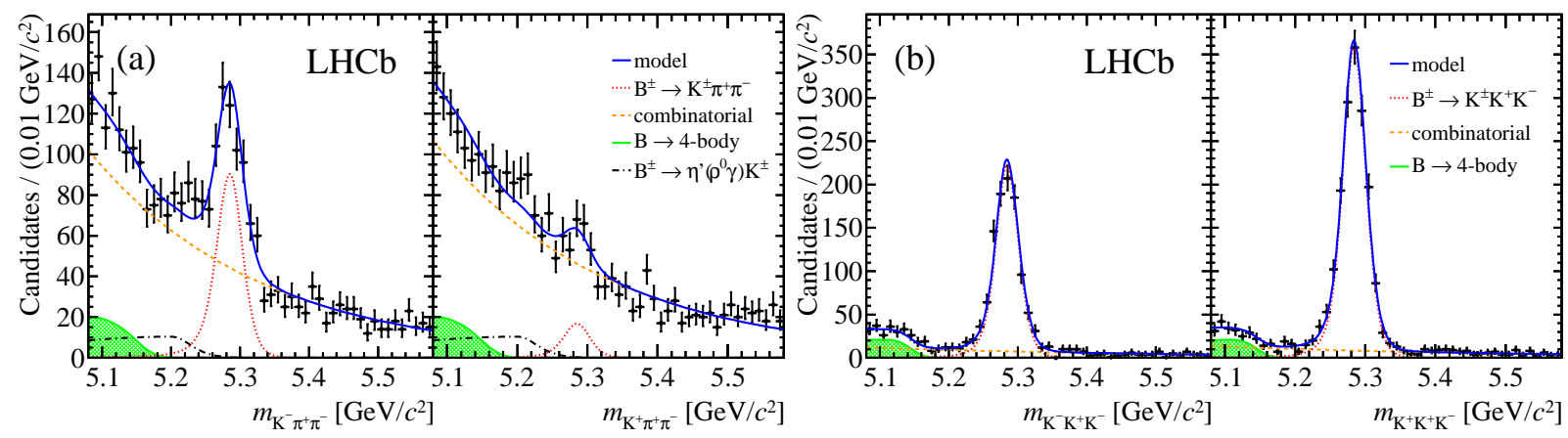

Figure 4: (a) Invariant mass reconstructed for $B^{+} \rightarrow K \pi \pi$ in the Dalitz region $0.8<m_{\pi \pi}^{2}<0.66 G e V^{2} / c^{4}$, and $m_{k \pi}^{2}<15 \mathrm{GeV}^{2} / \mathrm{c}^{4}$. (b) Invariant mass reconstructed for $B^{+} \rightarrow K K K$ in the Dalitz region $1.2<m_{K K l o w}^{2}<$ $2.0 \mathrm{GeV}^{2} / \mathrm{c}^{4}$ and $m_{K K h i g h}^{2}<15 \mathrm{GeV}^{2} / \mathrm{c}^{4}$. For both (a) and (b), the plot on the left is for $B^{-}$decay modes and the plot on the right is for $B^{+}$decay modes.

$\mathrm{CP}$ asymmetry along with the instrumental and production asymmetries. The instrumental asymmetry is determined from $D^{*}$ control samples in data and the production asymmetry is determined from time dependent decay rate spectra of $B^{0}$ and $B_{s}$. The CP asymmetries obtained are quoted below.

$A_{C P}\left(B^{0} \rightarrow K^{+} \pi^{-}\right)=-0.080 \pm 0.007$ (stat) \pm 0.003 (syst)

$A_{C P}\left(B_{s} \rightarrow K^{-} \pi^{+}\right)=0.27 \pm 0.04($ stat $) \pm 0.01$ (syst)

This is the first measurement of the direct CP violation in $B_{s}$ system. For the $B^{0}$ system, this is the most precise measurement to date. All these measurements are in agreement with the corresponding predictions from the standard model. The methods developed for this measurement were also used for measuring $\mathrm{CP}$ asymmetry in $B^{+} \rightarrow K \pi \pi$ and $B^{+} \rightarrow K K K$ channels.

\subsubsection{CP asymmetry in the $B^{+} \rightarrow K \pi \pi$ and $B^{+} \rightarrow K K K$ channels}

Using the 2011 data, LHCb measured the CP asymmetry in the $B^{+} \rightarrow K \pi \pi$ and $B^{+} \rightarrow K K K$ channels. The CP asymmetries obtained are quoted below [11] .

$A_{C P}\left(B^{+} \rightarrow K \pi \pi\right)=0.032 \pm 0.008($ stat $) \pm 0.004($ syst $) \pm 0.007$

$A_{C P}\left(B^{+} \rightarrow K K K\right)=-0.043 \pm 0.009$ (stat) \pm 0.003 (syst) \pm 0.007

Here the last term is from the systematic uncertainty related to the CP asymmetry in $B^{ \pm} \rightarrow J / \psi K^{ \pm}$ that was used as a control channel for this measurement.

This analysis was further refined by making these measurements in bins of the background subtracted Dalitz plot [11]. Using this it was found that for the $B^{+} \rightarrow K \pi \pi$ channel, in the region $0.8<$ $m_{\pi \pi}^{2}<0.66 \mathrm{GeV}^{2} / \mathrm{c}^{4}$, and $m_{k \pi}^{2}<15 \mathrm{GeV}^{2} / c^{4}$ the $\mathrm{CP}$ asymmetry is $0.678 \pm 0.078 \pm 0.032 \pm 0.007$ which is a $8 \sigma$ measurement. Similarly in the $B^{+} \rightarrow K K K$ channel, in a limited region of the Dalitz space $\left(1.2<m_{\text {KKlow }}^{2}<2.0 \mathrm{GeV}^{2} / \mathrm{c}^{4}\right.$ and $m_{\text {KKhigh }}^{2}<15 \mathrm{GeV}^{2} / \mathrm{c}^{4}$ where $\left.m_{\text {KKlow }}^{2}<m_{\text {KKhigh }}^{2}\right)$, the CP asymmetry is measured to be $-0.226 \pm 0.020 \pm 0.004 \pm 0.007$. This is a $10.5 \sigma$ measurement of $\mathrm{CP}$ asymmetry. For these channels, the reconstructed invariant mass of the $B^{+}$and that of the $B^{-}$ in the selected Dalitz region can be seen in Figure 4. In this figure, the difference in yields between the $B^{+}$and $B^{-}$, resulting from the corresponding CP asymmetry can be clearly seen. 


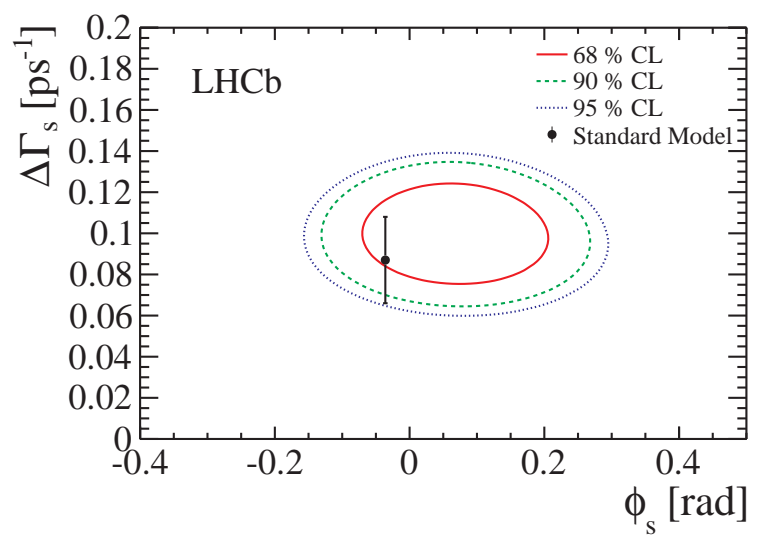

Figure 5: Two-dimensional profile likelihood in the $\left(\Delta_{\Gamma_{s}}, \phi_{s}\right)$ plane for the $B_{s} \rightarrow J / \psi K^{+} K^{-}$data. Only the statistical uncertainty is included. The standard model prediction of $\left(\Delta \Gamma_{s}=0.082 \pm 0.021 \mathrm{ps}^{-1}\right.$ and $\left.\phi_{s}=-0.036 \pm 0.002 \mathrm{rad}\right)$ is shown as the black point with error bar.

\subsection{CP violation in $B_{s} \rightarrow J / \psi K^{+} K^{-}$and $B_{s} \rightarrow J / \psi \pi^{+} \pi^{-}$}

In these channels $\mathrm{CP}$ violation occurs due to the interference between the mixing and decay into the same final state. Using the whole data set from 2011, LHCb measured [12] the mixing phase $\left(\phi_{s}\right)$, the mean width $\left(\Gamma_{s}\right)$ and the width difference $\left(\Delta \Gamma_{s}\right)$. The mixing phase $\left(\phi_{s}\right)$ is potentially sensitive to physics beyond the standard model. The $B_{s} \rightarrow J / \psi K^{+} K^{-}$proceeds via $B_{s} \rightarrow J / \psi \phi$, $\phi \rightarrow K^{+} K^{-}$(P wave) and the non-resonant $\mathrm{S}$-wave. The $\mathrm{P}$-wave is a mixture of $\mathrm{CP}$ even and $\mathrm{CP}$ odd and the $\mathrm{S}$ wave is purely $\mathrm{CP}$ odd. These are disentangled using the distribution of decay angles of the final state particles, defined in the helicity basis. The corresponding decay rate equations have a total of 10 terms. In the case of $B_{s} \rightarrow J / \psi \pi \pi$, the decay is mainly $\mathrm{S}$ wave and the decay rate equation has only 1 term. A maximum likelihood fit is made to the mass and the decay time to extract $\phi_{s}, \Gamma_{s}$ and $\Delta \Gamma_{s}$. As an example, in Figure 1 the distributions of the invariant mass and the decay time are shown. The result of the fit for $B_{s} \rightarrow J / \psi K^{+} K^{-}$can be seen in the plane of $\Delta \Gamma_{s}$ and $\phi_{s}$ in Figure 5. The results obtained after combining the two channels are quoted below.

$$
\phi_{s}=0.01 \pm 0.07(\text { stat }) \pm 0.01(\text { syst }) \mathrm{rad}
$$

$\Gamma_{s}=0.661 \pm 0.004($ stat $) \pm 0.006($ syst $) p^{-1}$

$\Delta \Gamma_{s}=0.106 \pm 0.011($ stat $) \pm 0.007($ syst $) p^{-1}$

These are compatible with the expectations from standard model and are the most precise measurement of these parameters to date.

\subsection{Measurement of CKM angle $\gamma$}

\subsubsection{ADS and GLW methods}

LHCb plans to use different methods to measure $\gamma$ using the 2011 and 2012 data. In the charged B decays, for example in $B^{+} \rightarrow \overline{D^{0}} K^{+}$and $B^{+} \rightarrow D^{0} K^{+}$for the case where the $D^{0}$ decays into a CP eigenstate $\left(K^{+} K^{-}\right.$or $\left.\pi^{+} \pi^{-}\right)$, the GLW [13] method is used. For the same $B^{+}$decay, for the case where the $D^{0}$ decays into $K^{+} \pi^{-}$or $K^{-} \pi^{+}$, the ADS [14] method is used. When using these methods with the different decay mode combinations and charge conjugate states, there are 

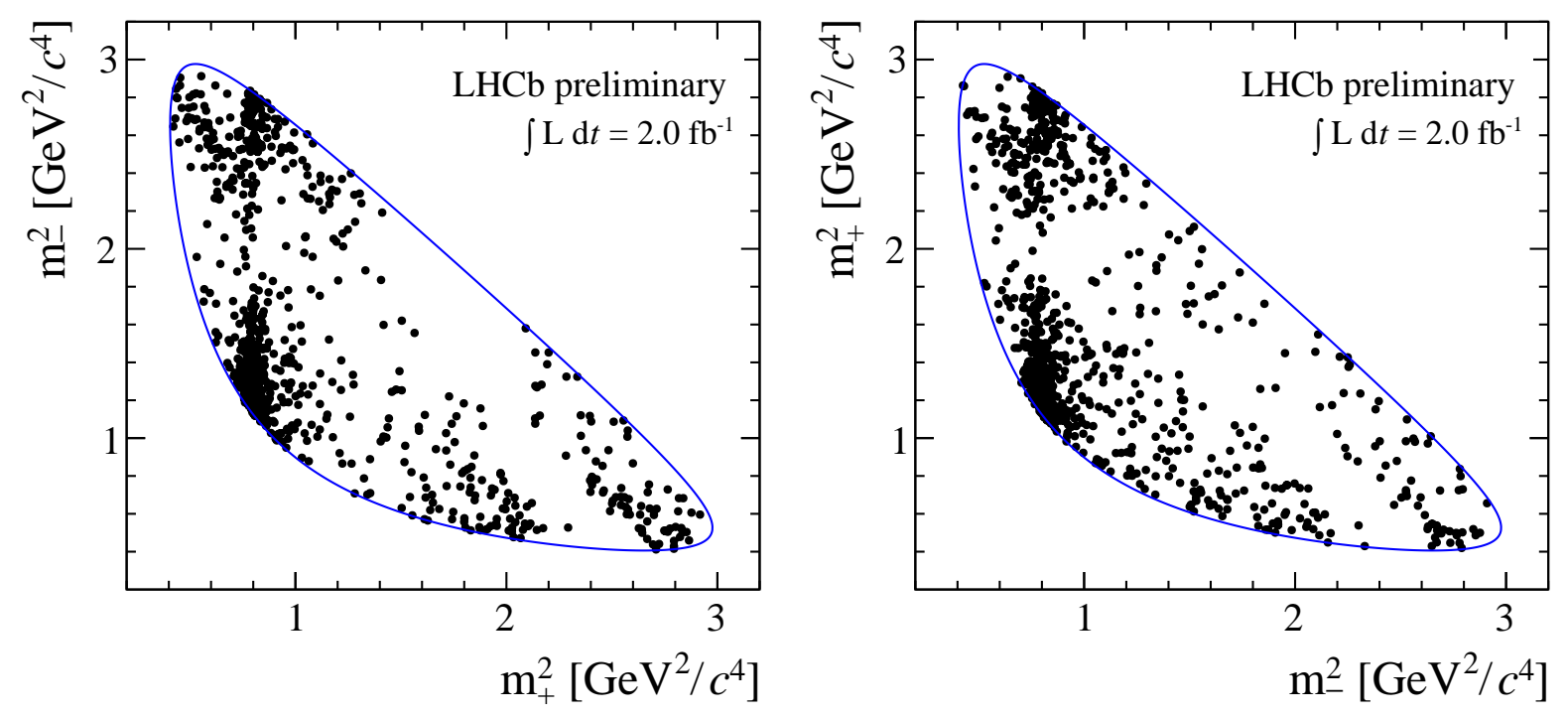

Figure 6: Left : Dalitz plot for the decay $B^{+} \rightarrow D^{0} K^{+}$where $D^{0} \rightarrow K_{s} \pi^{+} \pi^{-}$. Right: Dalitz plot for the decay $B^{-} \rightarrow D^{0} K^{-}$where $D^{o} \rightarrow K_{s} \pi^{+} \pi^{-}$.

16 data samples to fit for 13 observables. So far with the 2011 data, only the $\mathrm{CP}$ asymmetries and the ratios of partial widths between different modes are measured. These asymmetries are related to the angle $\gamma$ as indicated in [13] and [14].

Using the ADS mode, the CP asymmetry observed is at the $2.4 \sigma$ level in $B^{+} \rightarrow \overline{D^{0}} \pi^{+}$and is at $4.0 \sigma$ level in $B^{+} \rightarrow \overline{D^{0}} K^{+}$. Using the GLW method in different modes, a combined CP asymmetry at $4.5 \sigma$ level is observed [15]. Similar measurements are made also in the suppressed ADS mode $B^{+} \rightarrow D(\pi K \pi \pi) h$ where $\mathrm{h}=\mathrm{K}$ or $\pi[16]$.

\subsubsection{GCSZ method}

In the channel $B^{+} \rightarrow D^{0} K^{+}$where $D^{0} \rightarrow K_{S} \pi^{+} \pi^{-}$or $D^{0} \rightarrow K_{S} K^{+} K^{-}$, the GGSZ method [17] is used which makes use of a binned Dalitz analysis. In this method for each Dalitz bin $\left(m_{K_{s} h^{+}}^{2}\right.$, $m_{K_{s} h^{-}}^{2}$ ) the amplitude is expressed in terms of the CKM angle $\gamma$, the relative strong phase $\delta$ and the relative amplitude $r_{B}$ as described in [18]. Mass fits are made to the candidates in each Dalitz bin to extract the observables, $x_{ \pm}=r_{B} \cos \left(\delta_{B}+\gamma\right)$ and $y_{ \pm}=r_{B} \sin \left(\delta_{B}+\gamma\right)$. The Dalitz plots obtained for the $B^{ \pm} \rightarrow D K^{ \pm}$where $D \rightarrow K_{s} \pi^{+} \pi^{-}$, is shown in Figure 6. The results obtained [17] are $x_{+}=(-8.7 \pm 3.1 \pm 1.6 \pm 0.6) \times 10^{-2}, x_{-}=(5.3 \pm 3.2 \pm 0.9 \pm 0.9) \times 10^{-2}, y_{+}=(0.1 \pm 3.6 \pm 1.4 \pm$ $1.9) \times 10^{-2}$ and $y_{-}=(9.9 \pm 3.6 \pm 2.2 \pm 1.6) \times 10^{-2}$. In these results, the last term is the error on the strong phase measurement obtained from the CLEO experiment.

\subsubsection{Combined measurement of $\gamma$}

LHCb combined the results from all different methods into a log-likelihood fit to extract the $\gamma$. Figure 7 shows the plot of Gamma obtained from this procedure. The current value of $\gamma$ obtained from this procedure [19] is $\gamma=(67 \pm 12)^{\circ} @ 68 \%$ CL. 


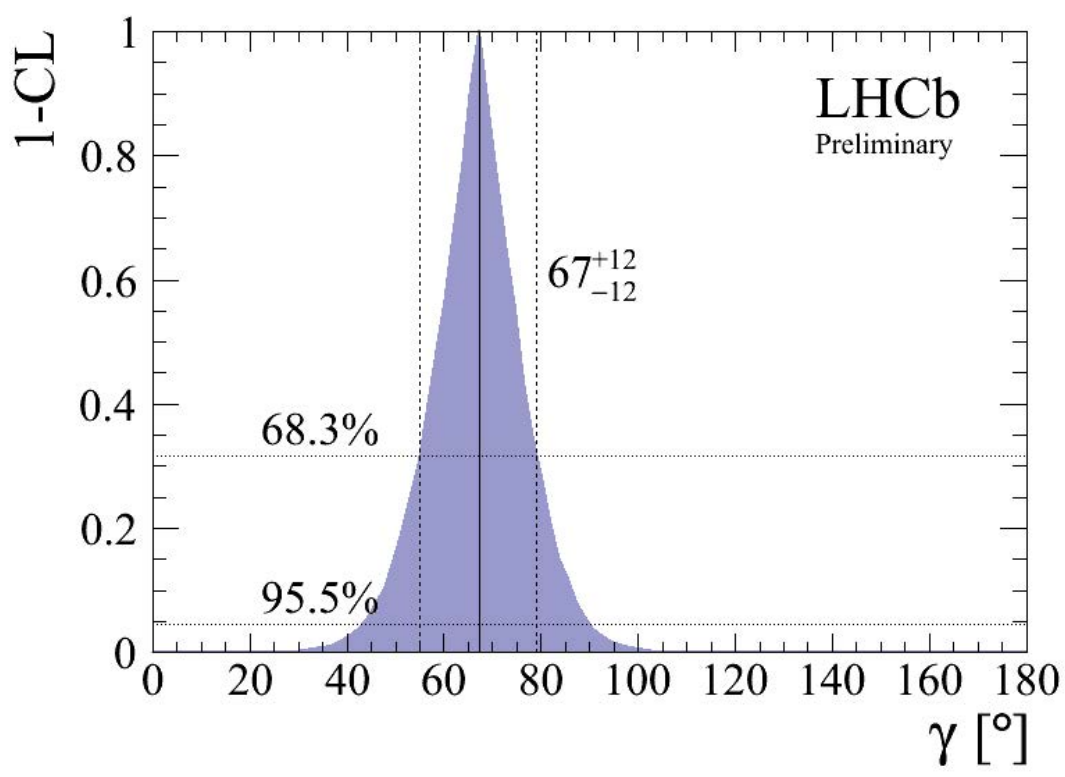

Figure 7: CKM angle $\gamma$ after the combined fit

\section{Results on $\mathrm{CP}$ violation and mixing in charm decays}

\section{1 $\Delta A_{C P}$ measurement}

The decay time dependent CP asymmetry $A_{C P}(f, t)$ for $D^{0}$ decaying into a CP eigenstate $f$ is defined as

$A_{C P}(f, t)=\frac{\left(\Gamma\left(D^{0}(t) \rightarrow f\right)-\Gamma\left(\overline{D^{0}}(t) \rightarrow f\right)\right)}{\left(\Gamma\left(D^{0}(t) \rightarrow f\right)+\Gamma\left(\overline{D^{0}}(t) \rightarrow f\right)\right)}$

This can be approximated to first order as $A_{C P}(f, t)=a_{C P}^{d i r}+(<t>/ \tau) a_{C P}^{i n d}$

Here $a_{C P}^{d i r}$ is the direct CP asymmetry, $a_{C P}^{\text {ind }}$ is the indirect CP asymmetry and $\tau$ is the $D^{0}$ lifetime. In the channel $D^{*} \rightarrow D^{0} \pi$ followed by $D^{0} \rightarrow f$ where $f=\left(K^{+} K^{-}\right.$or $\left.\pi^{+} \pi^{-}\right)$, the difference in time integrated $\mathrm{CP}$ asymmetry can be written as

$\Delta A_{C P}=A_{C P}^{d i r}\left(K^{+} K^{-}\right)-A_{C P}^{d i r}\left(\pi^{+} \pi^{-}\right)+(\Delta<t>/ \tau) a_{C P}^{\text {ind }}$

The measured asymmetry is $A_{\text {raw }}=A_{C P}+A_{P}+A_{D}$

Here the production asymmetry $\left(A_{P}\right)$ and the detection asymmetry $\left(A_{D}\right)$ cancels out when $\triangle A_{C P}$ is calculated. Using the data from 2011, LHCb measured the $\Delta A_{C P}$ in Prompt $D^{*}$ decays [20] and semi-leptonic B-decays [21].

For the $D^{*}$ analysis one can define the parameter

$\delta m=m\left(h^{+} h^{-} \pi^{+}\right)-m\left(h^{+} h^{-}\right)-m\left(h^{+}\right)$where $m$ is the mass and $\mathrm{h}=\mathrm{K}$ or $\pi$. This parameter is plotted in Figure 8 for the events after the selection. The KK and $\pi \pi$ mass obtained from the semileptonic analysis after the selections are shown in Figure 9. These figures show that clean signals were obtained for both these channels. From the $D^{*}$ analysis, LHCb measured

$\frac{\Delta<t>}{\tau}=[11.9 \pm 0.13($ stat $) \pm 0.17($ syst $)] \%$

$\Delta A_{C P}=[-0.34 \pm 0.15($ stat $) \pm 0.10($ syst $)] \%$

From the the semi-leptonic analysis LHCb measured $\frac{\Delta<t>}{\tau}=[0.018 \pm 0.002($ stat $) \pm 0.007($ syst $)]$ 

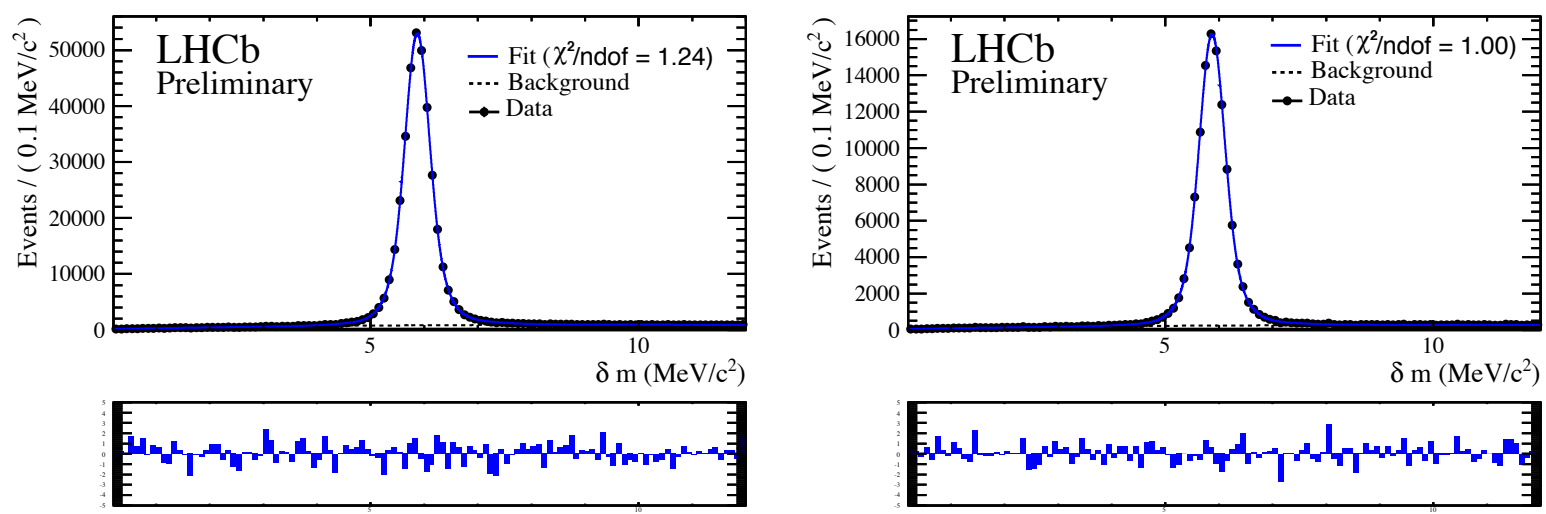

Figure 8: The $\delta \mathrm{m}$ parameter from the prompt $\mathrm{D}^{*}$ decays. Left: $\delta \mathrm{m}$ from the KK sample. Right $: \delta \mathrm{m}$ from the $\pi \pi$ sample
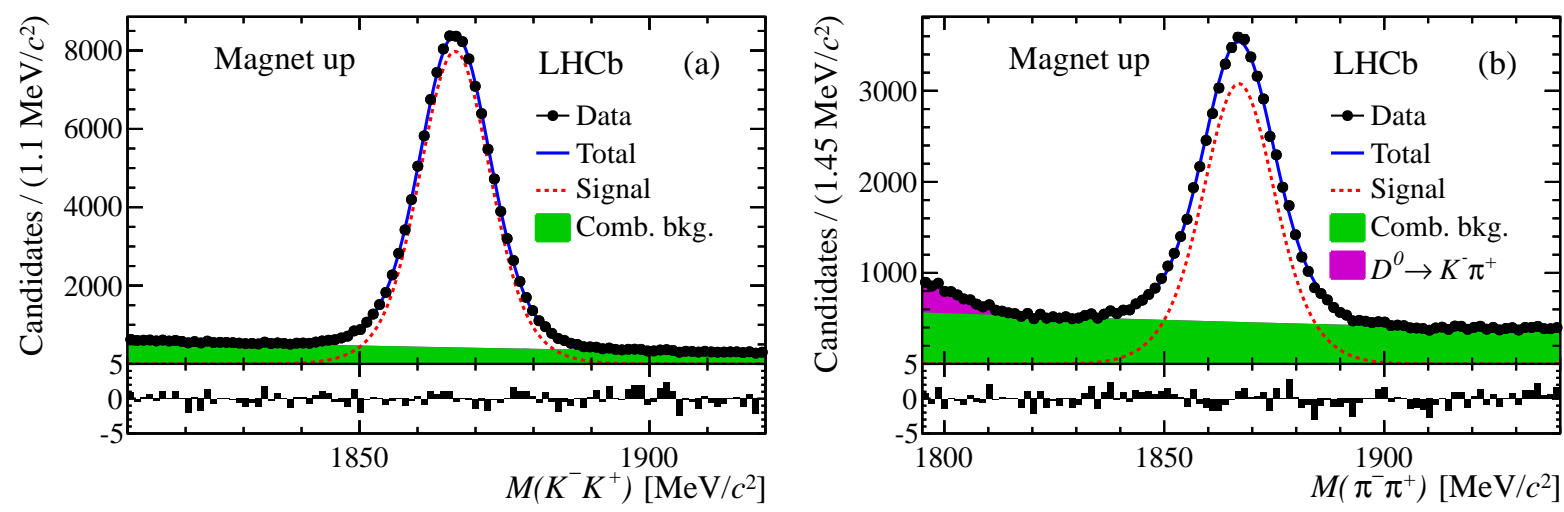

Figure 9: The reconstructed $D^{0}$ mass from the semi-leptonic B decays. Left: Invariant mass of the KK system . Right : Invariant mass of the $\pi \pi$ system

$\Delta A_{C P}=[0.49 \pm 0.30($ stat $) \pm 0.14($ syst $)] \%$

The difference between these two results is $2.2 \sigma$ and their weighted average is $\Delta A_{C P}=(-0.15 \pm$ $0.16) \%$. These results do not confirm evidence for direct CP violation in charm decays.

\subsection{Charm mixing}

Using the data from 2011, LHCb observed $D^{0}-\overline{D^{0}}$ oscillations by studying the time-dependent ratio of $D^{0} \rightarrow K^{+} \pi^{-}$to $D^{0} \rightarrow K^{-} \pi^{+}$decay rates [22] . The $D^{0}$ flavour at production is determined using the charge of the soft (low-momentum) pion $\pi^{+}$in the $D^{*+} \rightarrow D^{0} \pi^{+}$decay. The $D^{*+} \rightarrow D^{0}\left(\rightarrow K^{-} \pi^{+}\right) \pi^{+}$channel is dominated by a Cabibbo favoured (CF) amplitude and has a contribution from the mixing followed by double Cabibbo suppressed (DCS) amplitude. This channel is labeled as the right-sign (RS) events. The $D^{*+} \rightarrow D^{0}\left(\rightarrow K^{+} \pi^{-}\right) \pi^{+}$includes contributions from DCS amplitude and from mixing followed by Cabibbo favoured amplitude. This channel is labeled as the wrong-sign (WS) events. The mixing is characterized by two parameters, the mass difference $(\delta m)$ and the width difference $(\Delta \Gamma)$ between the two mass eigenstates of $D^{0}$. These are expressed in terms of the parameters $x, y$ where $x=\delta m / \Gamma$ and $y=\Delta \Gamma / 2 \Gamma$. In the limit of small 

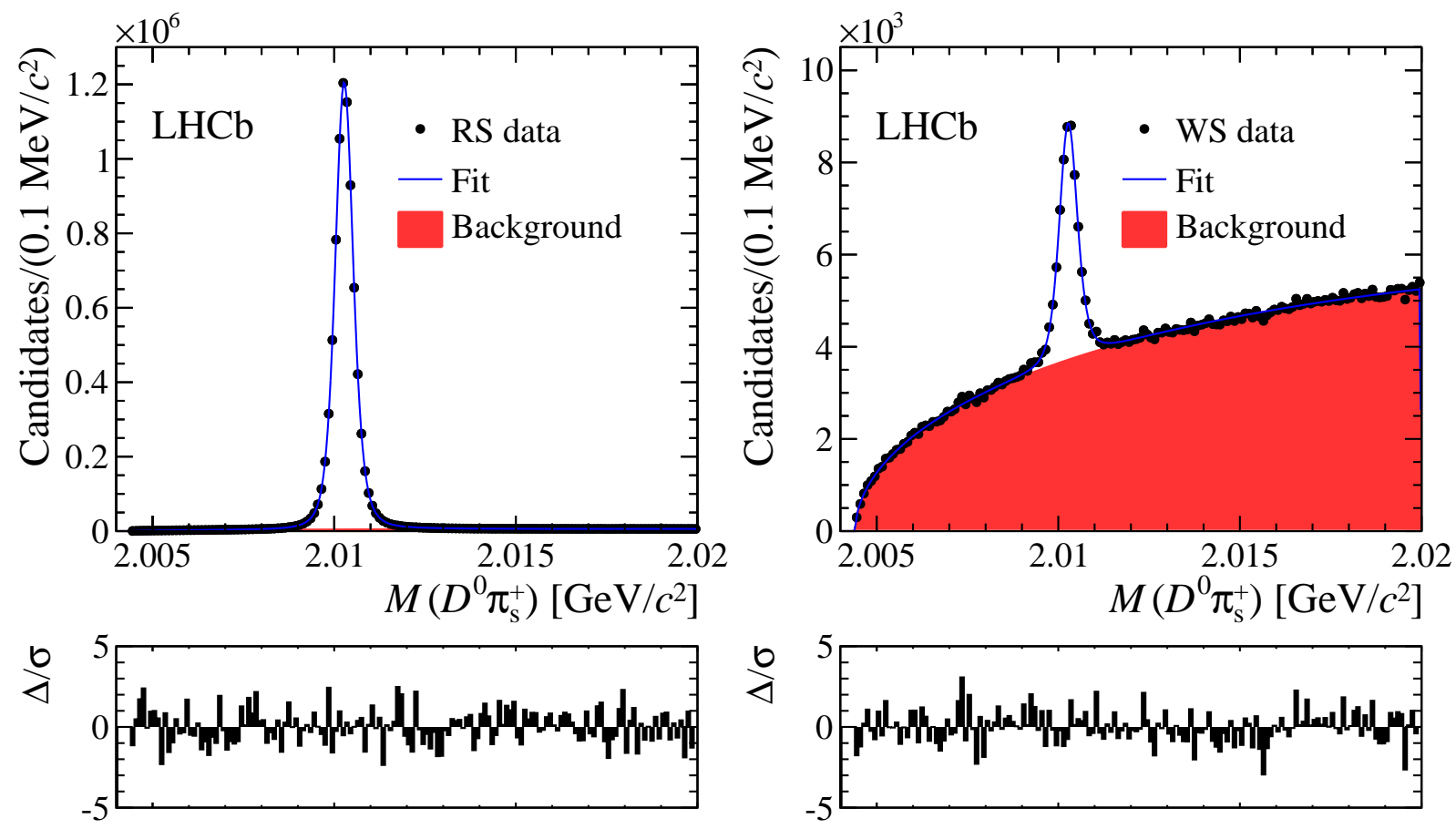

Figure 10: The reconstructed mass of the $D^{0} \pi$ system. Left: For the RS events, Right: For the WS events

mixing $(|x|,|y|<<1)$ and assuming negligible CP violation, the time dependent ratio (R) of WS to RS decay rate is approximated by the following equation. [22].

$R(t)=R_{D}+\sqrt{R_{D}} y^{\prime} \frac{t}{\tau}+\frac{x^{\prime 2}+y^{2}}{4}\left(\frac{t}{\tau}\right)^{2}$

here $\frac{t}{\tau}$ is the decay time expressed in units of the $D^{0}$ lifetime $\tau$. Also, $x^{\prime}=x \cos \delta+y \sin \delta, y^{\prime}=$ $y \cos \delta-x \sin \delta$ where $\delta$ is the strong phase difference between the DCS and CF amplitudes. The $R_{D}$ is the ratio of the DCS to CF decay rates. Figure 10 shows the invariant mass of the $D^{0} \pi$ for the RS and WS events. The background in the WS events is dominated by $\left(D^{0}+\right.$ random $\left.\pi\right)$ combinations. The data is divided into 13 bins of $(\mathrm{t} / \tau)$ and the ratio $(\mathrm{R})=\mathrm{WS} / \mathrm{RS}$ is determined for each bin and a $\chi^{2}$ minimization is used to extract the parameters $\left(R_{D}, x^{\prime 2}\right.$ and $\left.y^{\prime 2}\right)$. Figure 11 shows the result of this fitting for mixing and no-mixing hypothesis. The no-mixing hypothesis is excluded at $9.1 \sigma$. For the mixing hypothesis, the parameters obtained are listed below.

$R_{D}=(3.52 \pm 0.15) \times 10^{-3}$

$y^{\prime}=(7.2 \pm 2.4) \times 10^{-3}$

$x^{\prime 2}=(-0.09 \pm 0.13) \times 10^{-3}$

These are the most precise measurements of these quantities to date.

\section{Prospects for LHCb upgrade}

It is planned that, from 2019 onwards the luminosity in $\mathrm{LHCb}$ will increase up to $2.0 \times 10^{33} \mathrm{~cm}^{-2} \mathrm{~s}^{-1}$ at a collision energy of $14 \mathrm{TeV}$. With this LHCb is expected to collect a total of $50 \mathrm{fb}^{-1}$ data over several years, which would enable it to make measurements in different channels to reach sensitivities better than theoretical uncertainties. There is a large list of physics channels that can be studied 


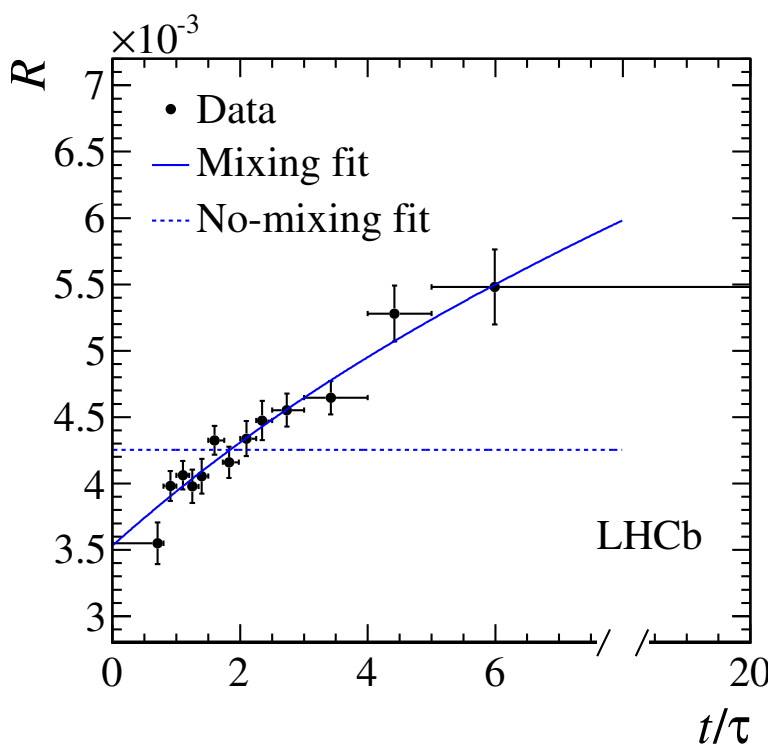

Figure 11: The ratio of WS/RS in each bin of the $(t / \tau)$. The fit is made for the mixing and no-mixing hypothesis.

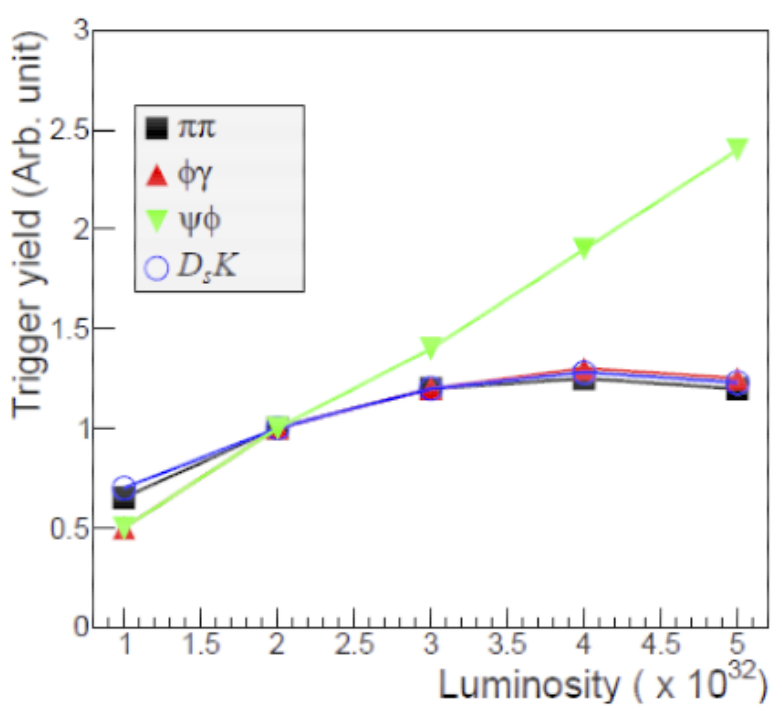

Figure 12: Trigger yield versus luminosity from current LHCb. At high luminosity, the leptonic trigger yield is not saturated, bu the hadronic triggers are saturated.

using the data from the upgraded detector and they give sensitivity to several key observables [23]. The current trigger scheme in LHCb has two limitations at these high luminosities. The front-end readout time is only about $1 \mathrm{MHz}$ and the limited discrimination power of the hadronic trigger causes the trigger yield to saturate at high luminosities. This can be seen from Figure 12 where the trigger yields at different luminosities are plotted.

The proposed solution [24] for this limitation is to read out the whole detector at $40 \mathrm{MHz}$ and 
use only software triggers. This requires an upgrade of the readout of all the detectors in LHCb. In addition to this, the vertex detector will use silicon pixel technology and the RICH detector will use new versions of multianode photomultipliers. The downstream tracking system is expected to use scintillation fibre technology.

\section{Summary}

The results from the data in 2011-12 show that the performances of the LHCb detectors were excellent during this period. LHCb has published several physics analysis results, some of which are described in this paper. In addition to the testing of the standard model with unprecedented precision, LHCb has started to search for new physics. It has excellent prospects to enhance its discovery potential in the coming years. An active upgrade program to run at the high luminosities from 2019, has started in LHCb.

\section{References}

[1] The LHCb Collaboration, R.Aaij et.al., Absolute luminosity measurements with the LHCb detector at the LHC , J.Instrum. 7 (2012) P01010

[2] The LHCb Collaboration, R.Aaij et.al.,Measurement of $\sigma(p p \rightarrow b \bar{b} X)$ at $\sqrt{s}=7 \mathrm{TeV}$ in the forward region., Phys. Lett. B 894 (2010) 209

[3] The LHCb Collaboration, R.Aaij et.al.,Prompt charm production in pp collisions at $\sqrt{s}=7 \mathrm{TeV}$, Nucl. Phys. B 871 (2013) 1

[4] N.Watson on behalf of the LHCb collaboration, Rare decays in LHCb , QFTHEP-2013 proceedings

[5] The LHCb Collaboration, A Augusto Alves Jr et.al.,The LHCb Detector at the LHC, J.Instrum. 01 (2008) S08005

[6] The LHCb RICH collaboration, Adinolfi,M et.al., Performance of the LHCb RICH detector at the LHC, Eur.Phys. J.C. 73 (2013) 2431

[7] A.A.Alves Jr et.al., Performance of the LHCb muon system, J.Instrum. 8 (2013) P02022

[8] R.Aaij et.al., The LHCb trigger and its performance, J.Instrum. 8 (2013) $\mathrm{P04022}$

[9] The LHCb Collaboration, R.Aaij et.al., Precision measurement of the $B_{s}^{0}-\overline{B_{s}^{0}}$ oscillation frequency with the decay $B_{s} \rightarrow D_{s}^{-} \pi^{+}$, New Journal of Physics 15 (2013) 053021

[10] The LHCb Collaboration, R.Aaij et.al., First Observation of CP Violation in the Decays of $B_{s}^{0}$ Mesons, Phys. Rev.Lett. 110 (2013) 221601

[11] The LHCb Collaboration, R.Aaij et.al., Measurement of CP violation in $B^{ \pm} \rightarrow K^{ \pm} \pi^{+} \pi^{-}$and $B^{ \pm} \rightarrow K^{ \pm} K^{+} K^{-}$decays , Phys. Rev.Lett. 111 (2013) 101801

[12] The LHCb Collaboration, R.Aaij et.al., Measurement of CP violation and the $B_{s}^{0}$ meson decay width difference with $B_{s} \rightarrow J / \psi K^{+} K^{-}$and $B_{s} \rightarrow J / \psi \pi^{+} \pi^{-}$decays, Phys.Rev. $D 87$ (2013) 112010

[13] M. Gronau, D. Wyler, On determining a weak phase from charged $B$ decay asymmetries ,Phys. Lett. B 265 (1991) 172 
[14] D. Attwood, I.Dunietz,A. Soni, Improved methods for Observing CP Violation in $B^{+} \rightarrow K D$ and Measuring the CKM Phase $\gamma$, Phys. Rev.D 63 (2001) 036005

[15] The LHCb Collaboration, R.Aaij et.al., Observation of CP violation in $B^{+} \rightarrow D K^{+}$decays, Phys. Lett. B 712 (2012) 203

[16] The LHCb Collaboration, R.Aaij et.al., Observation of the suppressed ADS modes $B^{+} \rightarrow\left[\pi^{ \pm} K^{\mp} \pi^{+} \pi^{-}\right]_{D} K^{ \pm}$and $B^{+} \rightarrow\left[\pi^{ \pm} K^{\mp} \pi^{+} \pi^{-}\right]_{D} \pi^{ \pm}$, Phys. Lett. $B 723$ (2013) 44

[17] The LHCb Collaboration, R.Aaij et.al., Model-Independent measurement of CP violation parameters in $B^{ \pm} \rightarrow\left(K^{0}+s h^{+} h^{-}\right)_{D} K^{ \pm}$decays, 14th International Conference on B-Physics at Hadron Machines, Bologna, Italy 8-12 April 2013, LHCb-CONF-2013-004

[18] A. Giri,Y. Grossman,A. Soffer,J. Zupan, Determining $\gamma$ using $B^{ \pm} \rightarrow D K^{ \pm}$with multibody D decays, Phys. Rev. D 68 (2003) 054018

[19] The LHCb Collaboration, R.Aaij et.al., Improved constraints on the CKM angle $\gamma$ from $B^{ \pm} D K^{ \pm}$ decays including first results on 2012 data, 14th International Conference on B-Physics at Hadron Machines, Bologna, Italy 8-12 April 2013 , LHCb-CONF-2013-006

[20] The LHCb Collaboration, R.Aaij et.al., A search for time-integrated CP violation in $D^{0} \rightarrow K^{+} K^{-}$ and $D^{0} \rightarrow \pi^{+} \pi^{-}$decays, 48th Reccontres de Moriond on QCD and High Energy Interactions, La Thuile, Italy 9-16 March 2013 , LHCb-CONF-2013-003

[21] The LHCb Collaboration, R.Aaij et.al., Search for direct CP violation in $D^{0} \rightarrow h^{+} h^{-}$modes using semileptonic B decays, Phys. Lett. B 723 (2013) 33

[22] The LHCb Collaboration, R.Aaij et.al., Observation of $D^{0}-\overline{D^{0}}$ oscillations, Phys. Rev. lett. 110 (2013) 101802

[23] The LHCb Collaboration, R.Aaij et.al., Implications of LHCb measurements and future prospects , EPJ C 73 (2013) 2373 , arXiv:1208.3355[hep-ex]

[24] The LHCb Collaboration, R.Aaij et.al., Framework TDR for the LHCb Upgrade , CERN-LHCC 2012-007 